\title{
Treatment of patients with a pituitary adenoma: one clinican's experience
}

\author{
Mary Lee Vance, M.D. \\ Departments of Medicine and Neurological Surgery, University of Virginia Health System, \\ Charlottesville, Virginia
}

\begin{abstract}
The diagnosis and treatment of a patient with a pituitary adenoma or another type of pituitary lesion requires a team approach involving the neurosurgeon, endocrinologist, primary care physician, and, in some patients, an ophthalmologist and radiation therapist. The diagnosis of excessive pituitary hormone secretion and/or loss of pituitary function is based on biochemical findings and can usually be confirmed by performing blood tests, except for patients with suspected Cushing syndrome. The primary treatment of pituitary adenomas is resection, except in patients with a prolactin-producing tumor, who are most successfully treated medically with a dopamine agonist. In this article the author reviews the principles of diagnosis and treatments and offers recommendations for ideal patient care.
\end{abstract}

\section{KEY WORDS • pituitary adenoma • hypopituitarism • multidisciplinary approach • surgery - medical therapy}

Effective treatment of a patient with a pituitary adenoma or another type of pituitary lesion requires communication and cooperation among several medical specialists, including the primary care physician, neurosurgeon, endocrinologist or neuroendocrinologist, neuroradiologist, ophthalmologist, anesthesiologist, and radiation therapy physician. No one medical specialist has sufficient expertise to provide total care for such patients. It is not appropriate to perform surgery in patients without first obtaining a preoperative evaluation by an endocrinologist to assess pituitary function, the need for preoperative hormone replacement therapy, and the role of medical treatment should the patient have a prolactinoma. It is not acceptable to treat patients surgically and then refer them for endocrine evaluation. The pituitary gland is both an anatomical and functional organ and management of a pituitary lesion is not solely a surgical consideration.

The treatment of a patient with a pituitary adenoma begins with an accurate diagnosis, which is made by obtaining a thorough history and physical examination, reviewing MR images, determining whether there is hormone hypersecretion or hormone deficiency, and correlating the clinical, anatomical, and endocrine findings. Assessment of visual acuity, visual fields, and ocular motility is also necessary in a patient harboring a macroadenoma that approximates or compresses the optic chiasm or invades the cavernous sinus or sinuses. The fact that MR

\footnotetext{
Abbreviations used in this paper: $\mathrm{ACTH}=$ adrenocorticotropic hormone; FSH = follicle-stimulating hormone; $\mathrm{GH}=$ growth hormone; IGF-I insulin-like growth factor-I; LH = luteinizing hormone; $\mathrm{MR}=$ magnetic resonance; $\mathrm{PRL}=$ prolactin; $\mathrm{T}_{4}=$ thyroxine; TSH thyroid-stimulating hormone; UFC = urinary free cortisol.
}

imaging detects a small lesion in the pituitary-"incidentaloma"-in approximately $10 \%$ of healthy persons, that is, those with normal pituitary function, emphasizes the need for a comprehensive clinical and endocrinological evaluation lest inappropriate interventions be recommended.

\section{DIAGNOSIS}

\section{Clinical Considerations}

The most frequent effects of a sellar mass are visual loss (macroadenoma) and headache (macroadenoma or microadenoma). Although headache is a common occurrence and is often the reason for undergoing MR imaging, it is a misconception that a small lesion cannot cause headaches. The sellar space is quite small and any size of lesion may produce or exacerbate headaches. Unfortunately, there is no specific feature of the headache to indicate a pituitary lesion; patients may report frontal, temporal, or occipital pain, or pain behind an eye. In addition to an assessment for mass effect, common historical features of a pituitary lesion include decreased libido and/or erectile dysfunction in men, irregular menses or amenorrhea in premenopausal women, and fatigue (thyroid hormone, cortisol, GH deficiency or deficiencies). Patients with acromegaly experience symptoms of the disease for approximately 7 years before diagnosis; their history includes the enlargement of hands, feet, and facial bones; joint pain; sleep apnea; excessive sweating; and skin tags. These changes are gradual and thus their appearance may not be noticed by the patient or family members or may be attributed to "aging." Patients with Cushing syndrome usually expe- 
rience weight gain (except for a few patients who exercise vigorously and may not have a dramatic weight gain), fatigue, difficulty with sleeping, irritability, depression, memory loss, difficulty with concentrating, muscle weakness, bone fractures, or osteoporosis. Development of diabetes or worsening of diabetes control and development of hypertension or worsening of treated hypertension are common features in patients with Cushing syndrome.

Diabetes insipidus is rare in a patient with a pituitary adenoma; however, it may occur in patients with a craniopharyngioma, a Rathke cleft cyst, or an infiltrative disease (lymphocytic hypophysitis, sarcoidosis, lymphoma, or metastatic tumor) because these disorders often involve the pituitary stalk and/or the hypothalamus (the site of antidiuretic hormone [vasopressin] production). A history of frequent urination, particularly frequent nocturia (every 1-2 hours), in a patient with a pituitary lesion is clinically diagnostic. Occasionally, a formal water deprivation test with vasopressin administration is indicated if there is doubt about the diagnosis and the need for desmopressin acetate therapy. This test must be performed in a hospital for the patient's safety.

Many patients with a pituitary lesion appear healthy on physical examination. Exceptions include patients with acromegaly or Cushing syndrome and men with hypogonadism. Clinical features of acromegaly include frontal bossing; coarse facial features including an enlarged nose, lips, tongue, and jaw (prognathism); increased spacing between the teeth; large "beefy" hands and feet; sweaty palms; and skin tags. Clinical features of Cushing syndrome include facial plethora, supraclavicular fat deposition, posterior cervical fat, acanthosis nigricans, acne, hirsutism, thin skin, ecchymoses, and violaceous striae; however, the last symptom is present in only $50 \%$ of patients and absence of violaceous striae does not exclude the presence of Cushing syndrome. In advanced Cushing syndrome, muscle wasting of the upper arms and thighs is evident and patients may not be able to arise from a chair without using their arms to push upward. Men with hypogonadism may have soft, small testes, and diminished beard and body hair are indicative of a long-standing testosterone deficiency. Fine wrinkling of the facial skin is characteristic and is likely to be a result of both a testosterone and GH deficiency. Expressible galactorrhea may occur with an elevated serum level of PRL, but the absence of galactorrhea does not exclude the presence of hyperprolactinemia.

\section{Anatomical Considerations}

Magnetic resonance imaging findings often provide a "clue" to the type of lesion, but confirmation, of course, can only be provided biochemically or, in patients who do not harbor a prolactinoma, by pathological examination of the tissue with appropriate immunostaining. A hypotense lesion on a non-contrast-enhanced MR image that demonstrates less enhancement than normal pituitary tissue following Gd administration raises the suspicion of a pituitary adenoma. If there are also enlarged sinuses and a thickened diploë, this feature is suggestive of acromegaly. A diffusely enlarged, homogeneously enhancing gland that involves the pituitary stalk is often seen in patients with an infiltrative disease such as lymphocytic hypo- physitis, sarcoidosis, or metastatic carcinoma. Although large pituitary adenomas may invade the cavernous sinuses and encase the internal carotid arteries, they usually do not affect the size of the artery. Rather, a decreased carotid artery size may occur with a meningioma, a metastatic tumor, or, occasionally, a chordoma. Extensive infiltration of the clivus raises concern about a chordoma. Purely cystic lesions are seen in a patient with a Rathke cleft cyst or an arachnoid cyst. Pituitary adenomas and craniopharyngiomas may have both cystic and solid components; however, multiple cysts, particularly those having a suprasellar extension, are more suggestive of a craniopharyngioma. A signal void (a black area) in the sella raises the concern that there may be a cavernous-carotid aneurysm, and an arteriogram is required for confirmation.

\section{Endocrine Considerations}

Assessment of hormone hypersecretion is straightforward and can be conducted on an outpatient basis. With the exception of Cushing disease, the diagnosis can usually be made using blood tests. Table 1 provides the appropriate screening blood tests performed in all patients with a pituitary adenoma. These blood tests are usually adequate to diagnose all but Cushing disease.

Hyperprolactinemia. Does this condition represent a prolactinoma or is it secondary hyperprolactinemia? In a patient with a macroadenoma $(>10 \mathrm{~mm})$ and an elevated serum level of PRL, it is necessary to determine if the hyperprolactinemia is a secondary phenomenon caused by an interference with the transport of dopamine, an inhibitor of PRL secretion, through the pituitary stalk, or if the tumor is producing PRL. In a patient with a true macroprolactinoma, the serum level of PRL is usually 200 $\mathrm{ng} / \mathrm{ml}$ or greater. A problem with the PRL assay is that the upper limit of detection is usually between 180 to 200 $\mathrm{ng} / \mathrm{ml}$; if the sample is not diluted, the value may be misleadingly reported as " $200 \mathrm{ng} / \mathrm{ml}$ " when, in reality, with

TABLE 1

Screening blood tests performed in patients with a pituitary adenoma*

\begin{tabular}{|c|c|}
\hline Blood Test & Purpose \\
\hline PRL (w/ dilution) & $\begin{array}{l}\text { diagnose prolactinoma; dilutions needed to cor- } \\
\text { relate PRL level \& tumor size for accurate } \\
\text { diagnosis }\end{array}$ \\
\hline $\begin{array}{l}\text { IGF-I (reflects over- } \\
\quad \text { all GH production) }\end{array}$ & $\begin{array}{l}\text { diagnose acromegaly (randomly tested GH level } \\
\text { may be normal) }\end{array}$ \\
\hline ACTH & $\begin{array}{l}\text { diagnose adrenal insufficiency; ACTH-depen- } \\
\text { dent Cushing syndrome }\end{array}$ \\
\hline cortisol (8 a.m.) & $\begin{array}{l}\text { diagnose adrenal insufficiency; not adequate to } \\
\text { diagnose Cushing syndrome }\end{array}$ \\
\hline $\mathrm{T}_{4}$ & $\begin{array}{l}\text { diagnose hypothyroidism (TSH level may be } \\
\text { normal in secondary hypothyroidism) }\end{array}$ \\
\hline $\mathrm{TSH}$ & diagnose TSH-producing adenoma \\
\hline LH & diagnose gonadotroph adenoma (tumor marker) \\
\hline FSH & diagnose gonadotroph adenoma (tumor marker) \\
\hline$\alpha$-subunit & diagnose gonadotroph adenoma (tumor marker) \\
\hline $\begin{array}{l}\text { testosterone } \\
\text { (male patients) }\end{array}$ & diagnose hypogonadism \\
\hline
\end{tabular}

* These blood tests are usually adequate to diagnose all but Cushing disease. 
adequate dilution the actual level is $2000 \mathrm{ng} / \mathrm{ml}$ or much higher, thus resulting in an incorrect diagnosis of a nonfunctioning adenoma with secondary hyperprolactinemia. Because the first line of treatment for a prolactinoma is medical therapy with a dopamine agonist, a misdiagnosis and inappropriate surgery may later prove to be problematic.

Cushing Disease. The diagnosis of Cushing syndrome is made by measuring the 24-hour level of UFC or the elevated nighttime level of salivary cortisol. A randomly chosen serum level of cortisol, even if elevated, is not adequate to assess overall cortisol production because an elevated serum cortisol level may result from the stress of venipuncture. Elevated levels of 24-hour UFC or nighttime salivary cortisol in conjunction with a nonsuppressed or elevated serum level of ACTH requires further evaluation to determine the source of the $\mathrm{ACTH}$, a pituitary tumor or an ectopic neuroendocrine tumor that most commonly comes from the lung or pancreas. Traditionally, the high-dose dexamethasone suppression test has been used to discriminate between pituitary-dependent and ectopic ACTH-based Cushing syndrome. Unfortunately, this test produces a significant amount of false-positive and false-negative results. The most reliable test performed to distinguish between pituitary-dependent and ectopic ACTH-based Cushing syndrome is inferior petrosal sinus sampling with administration of corticotropin-releasing hormone. The accuracy of this test approaches $100 \%$. One cautionary note: an experienced interventional radiologist should perform the study because cannulation of the inferior petrosal sinuses may be difficult.

Acromegaly. An elevated serum level of IGF-I is adequate to diagnose a GH-producing adenoma, which results in acromegaly. The IGF-I is produced primarily by the liver in response to $\mathrm{GH}$ and is an overall indicator of $\mathrm{GH}$ production. A single $\mathrm{GH}$ level appears "normal" because this hormone is released in pulses, which vary considerable from minute to minute. A normal GH level does not exclude acromegaly. Approximately $20 \%$ of patients with acromegaly have a concomitant hypersecretion of PRL. One must obtain a serum level of IGF-I to exclude the presence of acromegaly in a patient with hyperprolactinemia because the primary treatments of these two disorders differ: medical therapy is the primary treatment for a prolactinoma and surgery is the first choice for acromegaly. Although most patients with acromegaly have obvious clinical features of excessive $\mathrm{GH}$ production (coarse facial features, prognathism, frontal bossing, enlarged hands and feet, skin tags, joint pain, and hyperhidrosis), patients in whom the onset of disease is recent may appear healthy and may be asymptomatic, thus emphasizing the importance of measuring the IGF-I level. The definitive test for acromegaly is an oral glucose test in which blood glucose and GH levels are measured every 30 minutes for 2 hours. Failure of the GH level to decline to lower than $1 \mathrm{ng} / \mathrm{ml}$ is diagnostic of acromegaly. This test is most useful when performed after surgery to assess the completeness of tumor removal and the need for additional therapy.

Nonfunctioning Adenoma. On histological examination, most nonfunctioning adenomas are classified as go- nadotrophic tumors. Nevertheless, only a minority of patients are found to have elevations of the gonadotrophic hormones, LH and FSH, and the $\alpha$-subunit. Preoperative serum levels of LH, FSH, and the $\alpha$-subunit may be a valuable tumor marker in the event one or more of these hormone concentrations is elevated. If the level of one or more of these hormones is elevated preoperatively, they should be measured after tumor removal to assess the outcome of surgery and should again be measured during long-term follow up to detect tumor recurrence.

Thyroid-Stimulating Hormone-Producing Adenoma. The rarest type of pituitary adenoma is the TSH-producing tumor, which causes hyperthyroidism. Clinical manifestations of hyperthyroidism include palpitations, tremor, weight loss, difficulty with sleeping, hyperdefecation, and sweating. Diagnostic test results are an elevated serum level of free $\mathrm{T}_{4}$ in the setting of an increased TSH or a nonsuppressed TSH level. This diagnosis is particularly important because of potential operative complications (arrhythmias) if the patient is not adequately treated medically before surgery.

Hypopituitarism. The diagnosis of pituitary deficiency can often be made by performing a single blood test and a clinical assessment for DI. Pituitary adenomas, Rathke cleft cyst, craniopharyngioma, and infiltrative lesions most frequently cause hypogonadism, especially if the lesion is large. A menstrual history in premenopausal women is sufficient to assess gonadal function. Men often report diminished libido and/or erectile dysfunction. A low serum level of testosterone in conjunction with a low or "normal" serum level of LH is diagnostic of secondary hypogonadism. A low morning serum level of cortisol is indicative of secondary adrenal insufficiency and the need for steroid replacement. Assessment of the ACTH reserve requires a stimulation test. Practically, this is usually done after surgery, but the patient should be placed on a glucocorticoid regimen of hydrocortisone/prednisone during and after surgery with a full evaluation scheduled for a later date. A low serum level of free $\mathrm{T}_{4}$ is adequate to diagnose secondary hypothyroidism (the TSH level is usually normal). If the serum level of free $\mathrm{T}_{4}$ is low, thyroid hormone replacement is indicated.

\section{TREATMENTS}

\section{Surgical Treatments}

The goals of surgery are to remove the tumor, relieve the mass effect, improve visual abnormalities, reduce hormone hypersecretion to normal levels, and preserve normal pituitary function. Removal of a pituitary lesion by an experienced pituitary neurosurgeon offers the best possibility of achieving these goals, which are most likely accomplished in a patient with a small lesion. In reality, surgery is very effective in relieving the mass effect; however, most patients harboring a large lesion will require additional treatment(s) to produce hormonal remission and prevent regrowth of residual tumor, and many will require hormone replacement therapy to reestablish a normal hormonal balance. 


\section{Medical Therapies}

Prolactinoma. The first line of treatment for a patient with a prolactinoma is oral administration of a dopamine agonist, such as cabergoline, bromocriptine, or pergolide. More than $90 \%$ of patients respond to this treatment with reduction in the serum PRL level and tumor shrinkage. Patients who do not respond to a dopamine agonist, have intolerable side effects, or prefer a one-time treatment are candidates for surgery. It is important to emphasize that, in patients with a large tumor and a markedly elevated serum PRL level $(>1000 \mathrm{ng} / \mathrm{ml})$, a reduction of PRL to normal levels may never be achieved using medical therapy; however, an elevated PRL per se is not harmful to the patient as long as an appropriate hormone replacement therapy is given. This is in contrast to patients with acromegaly or Cushing disease in which elevated GH/IGF-I or cortisol levels increase the risk of morbidity and premature death. The most important consideration in a patient who has a macroprolactinoma with visual loss is reduction of the tumor volume to decompress the optic chiasm. If a trial of medical therapy for 2 to 4 weeks does not improve the visual abnormality, surgical debulking is indicated.

Acromegaly. At diagnosis, approximately $60 \%$ of patients with a GH-producing tumor are found to harbor a macroadenoma that is often invasive, making its complete removal unlikely. For this reason, in patients who do not have a visual loss, medical therapy with a somatostatin analog has been recommended as primary therapy by some neuroendocrinologists to control or improve the complications of acromegaly (heart failure, sleep apnea, or diabetes mellitus). This opinion is controversial, with other neuroendocrinologists advocating surgical debulking with postoperative medical therapy and radiotherapy as adjunctive treatments. Authors of small, uncontrolled studies have asserted that preoperative medical therapy with a somatostatin analog may improve surgical outcome, but this hypothesis has not been tested rigorously in a randomized, prospective fashion. Such a study is currently being conducted at several US and European centers that have expertise in pituitary surgery and neuroendocrinology.

Three classes of drugs are used to treat acromegaly: dopamine agonists, somatostatin analogs, and a GH-receptor blocker. None of these drugs destroy the tumor, but they do control the problem. Dopamine agonists lower the IGF-I level to normal in less than $20 \%$ of patients. Somatostatin analogs (octreotide or lanreotide) are administered by injection (octreotide delivered subcutaneously three times daily; long-acting release octreotide delivered intramuscularly once per month; and lanreotide delivered intramuscularly, every 10-14 days). Approximately $90 \%$ of patients experience an improvement in symptoms and some reduction in their serum GH and IGF-I levels. Nevertheless, in only 40 to $60 \%$ of patients is there a reduction in IGF-I levels to normal for the patient's age (the desired goal). In approximately $30 \%$ of patients there is some decrease in tumor size, but the amount of tumor shrinkage is variable and not as dramatic as that seen with medical treatment of prolactinomas. A reduction in tumor volume that is less than $50 \%$ is usual and, thus, this treatment is not a suitable initial treatment in patients with visual loss. The GH receptor antagonist, pegvisomant, does not act on the pituitary tumor; it inhibits dimerization of the $\mathrm{GH}$ receptor and prevents generation of IGF-I (the effector of $\mathrm{GH}$ action). Up to $97 \%$ of patients treated with pegvisomant experience a reduction in IGF-I to an age-adjusted normal level. Approximately five of 150 patients treated with pegvisomant have experienced tumor enlargement while taking this drug, making regular MR imaging studies necessary. Two patients had abnormal levels of liver enzymes, which returned to normal after discontinuation of the drug; liver enzymes should be measured every month for the first 6 months after the patient begins the drug regimen and periodically thereafter.

Cushing Disease. There is no effective medical therapy to reduce pituitary ACTH production or decrease tumor size. Surgical removal of the lesion is the first choice of therapy. If the surgery is not successful, pituitary irradiation should be considered. Medical therapy in which the antifungal agent, ketoconazole, is administered in higher doses than used to treat a fungal infection reduces adrenal cortisol production, thus effecting control of hypercortisolism while awaiting the effect of radiation. Ketoconazole may cause elevation of serum liver enzymes (aspartate aminotransferase, alanine aminotransferase, and $\gamma$-glutamyl transferase) and, if unrecognized, liver failure. For this reason liver enzyme levels should be measured before beginning ketoconazole therapy and monitored at regular intervals. There is no one effective ketoconazole dose for every patient. A reasonable starting dose lies between 200 and $400 \mathrm{mg}$ twice daily; after 1 month of treatment the 24-hour UFC level should be measured to assess the effectiveness of the therapy. Some patients require as much as $600 \mathrm{mg}$ twice daily to reduce the 24-hour UFC level to normal. A cautionary note: a patient may develop symptoms of adrenal insufficiency and the serum cortisol level should be measured if the patient reports fatigue, light-headedness, dizziness, anorexia, abdominal pain, or vomiting. Long-term or life-long therapy with ketoconazole is not recommended because of the risk of liver impairment.

Nonfunctioning Adenoma Craniopharyngioma, Rathke Cleft Cyst, and Infiltrative Disease. There are no effective medical therapies for these disorders. Resection is the primary therapy, with postoperative radiotherapy indicated in patients with significant residual disease or recurrence. Because these lesions often cause loss of pituitary function, it is vital to identify the need for a glucocorticoid (steroid), thyroid hormone, and vasopressin (desmopressin acetate) replacement before surgery to minimize the risk of intraoperative and postoperative complications. Diabetes insipidus should be anticipated postoperatively if there is involvement of the pituitary stalk or the hypothalamus. The need for gonadal steroid and GH replacement therapy should be evaluated after surgery.

Thyroid-Stimulating Hormone-Producing Adenoma. Hyperthyroidism should be controlled before a patient undergoes surgery because cardiac arrhythmias may occur during induction of anesthesia or intraoperatively. The anesthesiologist should be informed that the patient has hyperthyroidism. Treatment with a beta-blocker before surgery will control the effects of excessive thyroid hormone and reduce the potential for arrhythmia. If the sur- 
gery is elective (that is, there is no visual loss), the patient should also be treated beforehand with an antithyroid drug (propylthiouracil or methimazole) to reduce thyroid hormone production. The somatostatin analogs provide a very effective medical therapy that suppresses TSH production, decreases tumor size, and decreases thyroid hormone production. Somatostatin analog therapy is a very effective alternative to surgery in selected patients, particularly if there is no visual compromise. If disease remission has not been achieved after surgery, a somatostatin analog should be administered to prevent the untoward consequences of hyperthyroidism, reduce the size of any residual tumor, and prevent tumor growth.

Hypopituitarism. Adrenal insufficiency, hypothyroidism, and DI must be identified and treated before surgery to reduce the risk of intraoperative and postoperative complications. Glucocorticoid (intravenous hydrocortisone or dexamethasone) administration at the time of surgery is a usual and appropriate neurosurgical practice (except in patients with Cushing disease who do not require an intraoperative steroid). Steroid administration protocols differ among pituitary centers, but the general principle is to give a stress dose of a glucocorticoid at the time of surgery and for at least 24 hours afterward. Because hospitalization for transsphenoidal surgery is short term (2-3 days), at discharge many patients are given oral steroid replacement therapy with plans for reevaluation after surgical recovery (usually 6-8 weeks postoperatively). In this situation the patient should be given a short-acting glucocorticoid, hydrocortisone, to minimize the suppression of endogenous ACTH. Typically, the hydrocortisone therapy is discontinued 2 days before the postoperative visit and the serum levels of cortisol and ACTH are measured. The definitive test of ACTH reserve is the insulin hypoglycemia test, in which the patient is given a small dose of short-acting insulin to lower the blood glucose level to 40 $\mathrm{mg} / \mathrm{dl}$ or less with sequential measurement of glucose and cortisol levels. The normal response is a serum cortisol of $18 \mu \mathrm{g} / \mathrm{dl}$ or greater. Many patients have a GH deficiency both before and after surgery, and the definitive test of $\mathrm{GH}$ deficiency is the $\mathrm{GH}$ response to insulin-induced hypoglycemia. Thus, this test serves to diagnose both an impaired ACTH reserve and a $\mathrm{GH}$ deficiency, and should be reserved for the postoperative evaluation. Growth hormone replacement is beneficial in improving body composition (increase in muscle mass, decrease in adipose mass), muscle strength, exercise endurance, serum lipid level, and the patient's quality of life.

\section{Radiation Treatment of the Pituitary}

Radiation treatment (conventional, stereotactic, or proton-beam) of the pituitary is most commonly used as an adjunctive treatment after incomplete tumor resection. In patients with a large tumor in whom the resection is incomplete, radiotherapy reduces the risk of residual tumor enlargement and offers the chance of permanent control of hormone hypersecretion. No form of radiation delivery is immediately effective to reduce hormone hypersecretion and patients should be treated medically to control the disorder. Treatments for acromegaly include a somatostatin analog (lamreotide or long-acting release Sandostatin) or the $\mathrm{GH}$ receptor antagonist, pegvisomant (Somavert). Pa- tients with persistent hyperprolactinemia should be treated with a dopamine agonist (cabergoline, bromocriptine, or pergolide). As noted earlier, there is no effective treatment to reduce ACTH production in patients with Cushing disease; however, medical therapy with ketoconazole is very effective in reducing adrenal cortisol production and controlling Cushing disease. In a patient treated medically to control the disease while awaiting the effects of radiation therapy, the medication should be discontinued periodically (usually every 6 months) to determine if the radiation treatment has become effective and to determine whether there is any new pituitary hormone deficiency.

\section{Long-Term Disease Management}

Even with successful treatment, there is a life-long risk of tumor recurrence, regardless of tumor type, on the order of 12 to $16 \%$ within 10 years after surgery. Thus, any patient with a pituitary tumor requires life-long follow-up review and appropriate endocrinological and anatomical studies. Symptomatic recurrence in a patient with a hypersecretory tumor is straightforward and is confirmed by measurement of the appropriate hormone followed by an MR imaging study. In patients with a nonsecretory tumor, MR imaging studies should be performed annually for the first 5 years after surgery. If there is no recurrence or growth of residual tumor, the neuroimaging interval may be increased to every 2 years. A pituitary adenoma may recur 20 or more years after surgery and, thus, all patients should be observed throughout life. Other long-term management issues include determining the ongoing need for hormone replacement therapy, particularly in patients who have received pituitary radiation treatment and regular monitoring for adequacy of therapy.

Suggestions for Management of a Pituitary Lesion. The era of performing surgery in and discharging the patient from neurosurgical care no longer exists. Optimal management of a patient with a pituitary lesion of any type requires ongoing follow up by the primary care physician, the endocrinologist, and the neurosurgeon. The pituitary gland is both an anatomical and functional organ and as such requires the expertise of a multidisciplinary approach. At the initial evaluation the patient should be informed of the need for coordinated care by the primary care physician, endocrinologist, and neurosurgeon. If there is a recurrence or significant growth of a residual lesion, neurosurgical intervention may again be indicated, emphasizing the need for ongoing communication and cooperation among the patient's physicians. All physicians involved in the patient's care should send copies of office notes and opinions to all other physicians involved in the patient's care. This is a standard of care that can only benefit the patient.

The following are practical suggestions for the examination and treatment of patients with a pituitary lesion.

A patient should be examined by an endocrinologist before surgery. This is necessary to establish the diagnosis and to provide necessary pre- and postoperative hormone replacement regimens.

In a patient with a very large, invasive lesion, the patient should be informed before surgery that additional treatment(s) may be required. Surgery is necessary to reduce tumor volume and any mass effect, but the probabil- 
ity of complete tumor removal is low. It is wise to prepare the patient for the need for postoperative medical and/or radiation therapy.

It is probably not judicious to tell the patient "I got it all" immediately after the operation. Postoperative endocrinological studies, pathological examination of the tissue, and postoperative MR imaging are the definitive measures of surgical outcome. At the postoperative visit the response is often quite negative when a patient is told that additional treatment(s) are necessary, even though the neurosurgeon had said, "I got it all." It is appropriate to tell a patient after surgery that as much of the tumor was removed as was safely possible and that additional treatment(s) may be necessary, depending on the results of the postoperative studies.

Fertility issues must be discussed before surgery is performed in men and women in whom future fertility may be desired. Fertility can never be guaranteed, but it is important to emphasize that even if postoperative hypogonadism occurs, there are medical treatments that can promote ovulation or spermatogenesis. Hypopituitarism does not preclude potential fertility with appropriate hormone therapy.

Fortunately, most neurosurgeons review MR imaging studies personally and, in reality, may be more expert in their interpretation than general radiologists. Unfortunately, most endocrinologists rely on the radiology report and may not actually review the films with an experienced neuroradiologist, presuming one is available. Thus, it is very important for the neurosurgeon to inform the patient and the physicians involved with the patient's care of the MR imaging findings, any changes over time, and the need for additional treatment, including another operation.

\section{CONCLUSIONS}

The diagnosis and treatment of a patient with a pituitary adenoma or other pituitary lesion most often requires surgical intervention. Although usually very effective, pituitary surgery is not like a cholecystectomy; the operation is not the end of the problem. Given the potential need for hormone treatment before and after surgery, additional treatment such as pituitary radiation therapy, and the risk for recurrence, patients with pituitary lesions require lifelong follow up by all physicians involved in their care. A coordinated, multidisciplinary approach to the care of these patients should result in successful care and optimal outcomes.

Manuscript received February 15, 2004.

Accepted in final form March 9, 2004.

Address reprint requests to: Mary Lee Vance, M.D., Departments of Medicine and Neurological Surgery, University of Virginia Health System, P.O. Box 800601 Charlottesville, Virginia 22908. email: MLV@hscmail.mcc.virginia.edu. 